\title{
Rechtsgeschichte
}

\section{Thomas Wetzstein}

\section{Kein Handbuchwissen}


gegen das Verfahren vorzubringen, sucht man in $\mathrm{XI} / \mathrm{I}_{4} 2$ vergebens (so jedoch 210). Schließlich gibt es dem Wortlaut dieses Briefes zufolge keinen Anhaltspunkt dafür, dass die Mitglieder des Domkapitels von Chartres ihre Einwände gegen die Einsetzung des belasteten Kämmerers Radulphus erst in Form eines vorgeschalteten Exzeptionsverfahrens vorgebracht hätten und anschließend ein eigenes, päpstlich delegiertes Inquisitionsverfahren als denuntiatores angestrengt hätten (so jedoch 273).

Die verarbeitete Materialfülle stellte den Autor somit ganz offensichtlich vor verständliche Probleme einer adäquaten inhaltlichen Auswertung. Die ausgewählte Quellengattung birgt dabei zahlreiche Probleme mit erheblichen Konsequenzen für die Interpretation der Resultate. So weiß der Autor selbst, dass schätzungsweise nur 20 Prozent der ausgehenden Briefe Innozenz' III. überhaupt registriert wurden - hier scheint der Optimismus, es handele sich gerade dabei um die "meisten wichtigen Briefe « (39), abgesehen von der verblüffend unbefangenen Verwendung des

\section{Kein Handbuchwissen*}

Ein Handbuch, so lehrt der Brockhaus, ist ein »zusammenfassendes Werk über eine Wissenschaft oder ein spezielles wissenschaftliches Gebiet, häufig mehrbändig «. Nachdem die Bollandisten bereits zu Beginn des I7. Jahrhunderts das gegen einen wissenschaftlichen Zugriff häufig widerborstige Feld der Hagiographie mit beachtlichen Erfolgen eifrig zu bestellen begannen und dabei die Kanonisation der Heiligen in die Betrachtung einbezogen, legte Benedikt XIV. wenige Jahre vor seiner $\mathrm{I} 740$ erfolgten Wahl
Ausdrucks »wichtig «, wenig angebracht. Noch weitaus schwerer wiegt aber, dass Hirte auf der Grundlage dieser Quellen natürlich nur jene Verfahren zu fassen bekommt, mit denen die Kurie in einem bestimmten Moment beschäftigt war gerade aber seine zentrale These, die Wurzel des Inquisitionsverfahrens sei in der kirchlichen Disziplinarpraxis der örtlichen Kirchen zu suchen (259), ist mit Papstbriefen nicht zu belegen, zumal die in anderen Neuerungen im kirchlichen Verfahrensrecht wie den articuli als Quelle hervorgetretene Verfahrenspraxis der italienischen Kommunen in dieser Arbeit gänzlich ausgeblendet bleibt. Es muss vor diesem Hintergrund weiterhin offenbleiben, ob Innozenz III. tatsächlich »mehr evolutionär denn revolutionär " auf die Entwicklung des Inquisitionsverfahrens einwirkte (294). Eine neue empirische Grundlage vermag diese Studie dazu - unabhängig von der wissenschaftlichen Produktivität einer solchen Fragestellung - jedenfalls kaum zu bieten.

Thomas Wetzstein

zum Papst ein vierbändiges Opus über dieses für die westliche Kirche charakteristische Verfahren der Etablierung approbierter Kulte vor, das nicht nur eine bis auf den heutigen Tag unüberbotene Summe seiner profunden, häufig empirisch gewonnenen Kenntnisse der Materie darstellt, sondern zum Zeitpunkt seines Erscheinens mit vollem Recht als »Handbuch « hätte bezeichnet werden können. In den seither vergangenen Jahrhunderten ist die Forschung zu diesem Bereich nicht stehen geblieben, wenn sie

\footnotetext{
* Otfried Krafft, Papsturkunde und Heiligsprechung. Die päpstlichen Kanonisationen vom Mittelalter bis zur Reformation. Ein Handbuch (Archiv für Diplomatik, Schriftgeschichte, Siegel- und Wappenkunde, Beiheft 9), Köln, Weimar, Wien: Böhlau 2005, XII, I 247 S., ISBN 3-4I 2-25805-9
} 
auch mittlerweile fast ausschließlich in die $\mathrm{Zu}$ ständigkeit der Historiker übergegangen ist. So könnte es an der Zeit sein, erneut einen Überblick über dieses Thema in Form eines Handbuchs vorzulegen: Otfried Krafft hat dies mit seiner 2003 bei Jürgen Petersohn in Marburg entstandenen historischen Dissertation laut Untertitel (»Die päpstlichen Kanonisationen vom Mittelalter bis zur Reformation «) unternommen. 64 zwischen den Jahren 993 und I 523 von den Nachfolgern Petri durchgeführte Kanonisationen hat sich der Verfasser vorgenommen, um die Einzelumstände der Verfahren, vor allem aber die Kanonisationsurkunden als das nach landläufiger Meinung wichtigste Ergebnis der sich häufig über Jahre erstreckenden Prozedur mit Hilfe der etablierten Methodik der Urkundenkritik in »Funktion und Formular « (I3) zu untersuchen und darzustellen.

Die Ergebnisse dieser über ıooo Seiten umfassenden, mit großem Fleiß und einer immer selteneren gewissenhaften Akribie erstellten Studie in wenigen Sätzen zusammenzufassen, dürfte nur eine Ahnung jener Schwierigkeiten bieten, die der Autor bei der Zusammenstellung und Auswertung seines geradezu erdrückenden $\mathrm{Ma}$ terials zu überwinden hatte und an welchen er den Leser gelegentlich bei der Lektüre der Fußnoten teilhaben lässt. Schon weil der Verfasser entlegenste Beiträge zu einzelnen Verfahren nahezu ohne Rücksicht auf Sprache und Erscheinungsjahr bequem zugänglich macht, verdient das Werk Anerkennung und Aufmerksamkeit. Die Bestände von 5I über Europa verstreuten Archiven und Bibliotheken gingen überdies in die Darstellung ein, und nur vor der explodierenden handschriftlichen Überlieferung des I 5. Jahrhunderts musste selbst Krafft kapitulieren und sich aus arbeitsökonomischen Gründen auf die Kanonisationsurkunden beschrän- ken und die zahlreichen begleitenden Texte wie päpstliche Ansprachen, Konsistorialreden, Briefwechsel, Zeremonialschriften und Prozessakten weitgehend außer Acht lassen (3, 940). Die Chronologie gab dem Verfasser die Gliederung vor, und so widmen sich I 5 der insgesamt I7 Kapitel den einzelnen, nach Zeiträumen und Päpsten gegliederten Kanonisationen von Ulrich von Augsburg (993) bis Benno von Meißen ( 1523 ), deren Ergebnisse am Ende jeweils knapp zusammengefasst präsentiert werden. Ein Schlusskapitel stellt die Resultate der Arbeit unter systematischen Gesichtspunkten vor.

Die Auskunftsfreude der sachgerecht analysierten Kanonisationsurkunden für übergreifende Fragestellungen vermag dabei in Erstaunen zu versetzen. So findet etwa die zunehmende Konzentration der Kirchenmacht auf die Person des Papstes auch in der Formularentwicklung beredten Ausdruck: Während die Urkunden der Frühzeit noch ganz den Charakter eines Synodaldekrets mit der Unterschrift der beteiligten Prälaten (55) besitzen und die fehlende Beteiligung eines Konzils am Kanonisationsentscheid noch in der Mitte des I2. Jahrhunderts eigens erwähnt und legitimiert werden muss (9I, I I I), tritt im Pontifikat Alexanders III. (I I 59-I I 8I) allein das Vertrauen des Papstes auf Gott und die Apostel an genau jene Stelle, an der vorher vom Konzil als kompetenter Instanz einer Kanonisation die Rede war (I I 8 f.). Erst das Konstanzer Konzil sollte einen freilich erfolglosen, aber gerade deswegen vielsagenden Versuch unternehmen, mit der eigenständigen Einleitung dreier Kanonisationsverfahren diese seit fast drei Jahrhunderten vollzogene Entwicklung umzukehren (863).

Wie wenig die Kanonisation der Heiligen dabei einheitlichen Prinzipien folgte, zeigt ein Detail der äußeren Merkmale von Kanonisa- 
tionsurkunden: Noch bis ins I5. Jahrhundert versah die päpstliche Kanzlei die Siegel dieser Schriftstücke teils mit einer Hanf-, teils mit einer Seidenschnur und ließ damit die Frage offen, ob Kanonisationen eher als päpstliches Mandat, durch welches ein Kult angeordnet wurde, oder als Privileg, das einen Kult gewährte, zu betrachten waren (I05I). Eine minutiöse Analyse der Adressaten vermochte in Kombination mit der teilweise erhaltenen Empfängerüberlieferung zu zeigen, dass die beständig gewachsene Kommunikationsmacht der Kurie nicht immer für Kanonisationen genutzt wurde, sondern vielmehr zwischen bewusster Mehrfachausfertigung oder Massenausfertigung (4I8) mit genauen Anweisungen zur Bekanntmachung des neuen Heiligenfestes in der Christenheit bis hinunter auf die Ebene der Pfarrei einerseits (130) und andererseits Einzelausfertigungen mit nur einem Adressaten oszillieren konnte (858) - ja, dass die Kanonisation eines Heiligen nicht nur umstritten sein (793), sondern im Extremfall gänzlich in Vergessenheit geraten konnte (919). Die Ursache dieses Sachverhalts ist nicht zuletzt darin zu sehen, dass auch die Päpste des Mittelalters das neue Instrument der Schriftlichkeit erst allmählich gezielt und systematisch einsetzten und noch bis weit ins I2. Jahrhundert Kanonisationen vornahmen, ohne darüber eine schriftliche Urkunde auszustellen (73). Auch die in späterer Zeit überwiegend schriftlich erteilten Anweisungen des Papstes zur örtlichen Untersuchung der einem Kandidaten zugeschriebenen Mirakel wurden erst unter Alexander III. in schriftlicher Form erteilt, und im selben Pontifikat erfolgte eine erste »Kanonisation nach Aktenlage « - auf der Grundlage eines schriftlichen Berichts der vor Ort entsandten Kardinäle ( I 22).

Die genaue Funktion der einzelnen Urkunden lässt sich, wie der Autor freimütig bekennt, nur unter Schwierigkeiten bestimmen (420) was im Besonderen für das Schicksal jener Ausfertigungen gilt, die nicht an den Kultort selbst, sondern unter Umständen in eine davon weit entfernte Region der lateinischen Christenheit gelangten. In einigen Fällen hingegen informieren narrative Quellen recht detailliert darüber, wie die Kanonisationsurkunde an die Begräbnisstätte des Heiligen gelangte und dort feierlich im Rahmen einer liturgischen Handlung verlesen wurde (I I 5), während eine im I 5. Jahrhundert gelegentlich nachweisbare bildliche Ausgestaltung des Kanonisationsdokuments darauf hinweisen dürfte, dass im späteren Mittelalter Kanonisationsurkunden ähnlich wie Reliquien auch ostendiert wurden ( $\mathrm{I} \circ \mathrm{O} 3$ ). Somit weist die Verwendung der Urkunden zumindest in Einzelfällen über ihren allgemeinen Gebrauch im Rahmen der Kultpflege, der Sicherung des rechtlichen Sachverhalts oder in ihrer Relevanz für juristische Fragen (I054) hinaus - was vor allem Benedikt XIV. zur Darstellung der von ihm postulierten einheitlichen Entwicklung des Verfahrens und der kurialen Rechtsgewohnheiten erkannte. Auch die Kurie selbst nutzte freilich wie in anderen der immer zahlreicher werdenden Rechtsbereiche das eigene institutionelle Gedächtnis und griff über Register oder eigene Brief- und Formelsammlungen auf bewährte Formulare zurück, wie dies Krafft anhand einer schematischen Übersicht im Anhang besonders gut zu zeigen vermag (I062-IO7I).

Gerade in der Behandlung der Kanonisationen durch die Kurie werden allerdings auch einige Schwächen der Studie sichtbar, die sich nicht zuletzt aus der ohnehin immer wieder durchbrochenen Konzentration des Autors auf die Kanonisationsurkunden ergeben. Sie entstehen nämlich jeweils in einem spezifischen und sich dynamisch wandelnden Kontext, der sich 
nicht immer authentisch in den Urkunden spiegelt. So fehlt etwa eine Darstellung der zugegebenermaßen komplexen historischen Entwicklung der päpstlichen Kanzlei völlig, so dass gelegentliche Hinweise auf Formelbücher (855) oder Tax- bzw. Kanzleivermerke (677, 862, 94I Fn. 13, 967, 987, 99 I Fn. 269) gänzlich in der Luft hängen, ja völlig unklar bleibt, wer denn für die Konzeption der ausführlich inhaltlich analysierten Kanonisationsurkunden überhaupt verantwortlich war und ob sich hinter dem im I 5. Jahrhundert gelegentlich nachweisbaren Registervermerk de curia tatsächlich ein Anliegen des Papstes » in eigener Sache « (958 Fn. 98) oder nicht vielmehr das erfolgreiche Bemühen der Petenten um eine Gratisexpedition verbirgt. Neuere Studien zum mittelalterlichen Urkundenwesen haben überhaupt den Anteil der Petenten immer stärker betont, und so wüsste man eben auch hier gerne, welcher Anteil im Formular der Urkunden dem Papst, der Kurie oder aber den Antragstellern - wie nur sporadisch vermerkt (I 50) - zuzuschreiben ist.

Dass diesen der maßgebliche Anteil bei Einleitung, Durchführung und erfolgreichem Abschluss in der Mehrzahl der Verfahren zukam, während Papst und Kurie kaum aktiv in den Verfahrensverlauf eingriffen, droht bei unvoreingenommener Lektüre des Werks aus dem Blick zu geraten. Es mag einer erfolgreichen Selbstdarstellung des Papsttums zuzuschreiben sein, dass Krafft nach Gründen sucht, die zum Scheitern mancher Verfahren führten $(855)$ - historisch angemessener wäre es, das Erklärungsbedürftige zu erklären. In der Gesamtbetrachtung nämlich erscheint das Misslingen eines Kanonisationsversuchs als Normalfall, während der seltene erfolgreiche Abschluss eines Verfahrens grundsätzlich aus den politischen Umständen, Einfluss- und Finanzmitteln der Antragsteller und ihrer Förderer oder spezifischen Interessen der Kurie zu begründen ist, wie dies der Autor in einigen Fällen unternommen hat. Welche Erfolgschancen einem Kanonisationsantrag im Vorfeld zuzubilligen waren, bereitete der Kurie nicht nur keine Sorgen (543) und unterstellt eine anachronistische Verfahrensrationalität - ein Kurialer hätte dazu kaum verlässlichere Aussagen machen können als ein DFG-Mitarbeiter zu den Aussichten auf die Einrichtung eines neuen Sonderforschungsbereichs.

Ebenso wenig wie der institutionelle Rahmen lässt sich darüber hinaus der verfahrensrechtliche Kontext aus der Beschäftigung mit der Kanonisation ausblenden. Dies gilt in besonderem Maße für das von Krafft ausführlich berücksichtigte I3. Jahrhundert, in welchem im Rahmen einer beispiellosen Verrechtlichungswelle der lateinischen Kirche nahezu alle an die Kurie herangetragenen Anliegen zunehmend nach den Regeln des gelehrten Prozessrechts behandelt wurden. Delegation und Subdelegation, kuriale Rechtsprechung und Aktenbearbeitung, prozessuale Stellvertretung und Protokollierungszwang, Frageartikel und Interrogatorien, Zeugenbeweis und Beweislast - all dies findet sich seitdem auch im Kanonisationsverfahren und fehlt weitgehend im vorliegenden Buch. So heißen die für den gelehrten Prozess typischen und zur Urteilsfindung herangezogenen Rubriken und Summarien bei Krafft schlicht "verschiedene Zusammenfassungen des Prozeßstoffes " (675), die charakteristische Anwendung der Verhandlungsmaxime erscheint als » beständiges Sollizitieren " ( 1033$)$ - das in Einzelfällen sogar so weit gehen konnte, dass die zuständigen Kardinäle den Antragstellern auferlegten, ihnen die Akten eines erfolgreichen Prozesses zur Verwendung als Modellverfahren zu beschaffen (955 Fn. 84) -, und das prozesseinleitende Ernen- 
nungsschreiben des Papstes an die delegierten Kommissare ist den Akten nicht nur »relativ oft inseriert « (IO54), sondern gehört nach den Regeln des römisch-kanonischen Prozesses zwangsläufig zu allen Akten delegierter Verfahren. Die dabei seit dem I 5. Jahrhundert zu beobachtende Praxis einer mündlichen Delegation des Verfahrens an Kardinäle (commissio vivae vocis oraculo) im Rahmen des Konsistoriums mit deren anschließender Subdelegation an Prälaten in partibus führt zur Einschätzung, dass sich die Verfahrenseinleitung »etwas vom Papst selbst entfernte « (I006), während dieses in anderen Konsistorialangelegenheiten ebenfalls übliche Verfahren zunächst als ein frühes Beispiel von Bürokratieabbau zu betrachten ist und die Bedeutung des Papstes in diesem entscheidenden Moment der Verfahren keineswegs verminderte.

Nur die Kenntnis der seit dem I3. Jahrhundert immer stärker verfestigten Verfahrensschritte hätte dem Autor Einblicke in bemerkenswerte Anomalien gewährt - so etwa bei Sixtus IV. (I47I-I484), der seinen eigenen Antrag auf die Kanonisation Bonaventuras genehmigte, oder bei Pius II. (I458-I464), der bei der Kanonisation der Katharina von Siena auf die Durchführung der sonst üblichen lokalen Untersuchung verzichtete und diese kurzerhand durch eine Jahrzehnte zurückliegende bischöfliche inquisitio ersetzte. Diese aber hatte nichts anderes als unerlaubte Praktiken des Katharinenkults zum Gegenstand gehabt. Legte man auch hier die strengen Kriterien Kraffts an, der beispielsweise eine Kanonisation für Sebald von Nürnberg (1425) deshalb ablehnt, weil kein eigenes Verfahren durchgeführt worden war und das Urkundenformular an einigen Stellen von den üblichen Formulierungen abwich (946 Fn. 33), so wären auch bei diesen weithin bekannten Fällen Zweifel angebracht, ob es sich um eine Kanonisation im engen Wortsinne handelte. Die mittelalterlichen Kanonisten hätten allerdings über eine solche Ansicht wohl den Kopf geschüttelt, galt ihnen doch der Papst als suprema potestas, die sich nicht selbst durch Verfahrensvorschriften binden, sondern von diesen unter Umständen durchaus abweichen konnte.

Die hier vorgetragenen Monita sollten allerdings nicht zur Überzeugung führen, das vorliegende Werk sei in seinen klar erkennbaren Grenzen nicht als entscheidender Beitrag zur Geschichte der Kanonisation im Mittelalter zu betrachten. Dafür bürgen schon allein der geschliffene und verständliche Stil, die Solidität der Darstellung und vor allem die Vielzahl der verwendeten Quellen. Eines aber dürfte nach den Hinweisen auf die Lücken der Studie allein für das I 5. Jahrhundert deutlich geworden sein: Auf ein neues Handbuch, das »die päpstlichen $\mathrm{Ka}$ nonisationen " zwischen 993 und 1523 im umfassenden Sinne des Eingangszitats behandelt, wird die Forschung weiter warten müssen.

Thomas Wetzstein 